\title{
Discrimination between Dementia Groups and Healthy Elderlies Using Scalp-Recorded-EEG-Based Brain Functional Connectivity Networks
}

\author{
Sakura Nishijima ${ }^{1}$, Tetsushi Yada ${ }^{1}$, Toshimasa Yamazaki ${ }^{1}$, Yoshiyuki Kuroiwa ${ }^{2}$, \\ Makoto Nakane ${ }^{3}$, Kimihiro Fujino ${ }^{2}$, Toshiaki Hirai'2, Yasuhisa Baba², Shoko Yamada ${ }^{3}$, \\ Sho Tsukiyama ${ }^{1}$
}

\begin{abstract}
${ }^{1}$ Department of Bioscience and Bioinformatics, Kyushu Institute of Technology, Fukuoka, Japan; ${ }^{2}$ Department of Neurology, University Hospital, Mizonokuchi Teikyo University School of Medicine, Kanagawa, Japan; ${ }^{3}$ Department of Neurosurgery, University Hospital, Mizonokuchi Teikyo University School of Medicine, Kanagawa, Japan
\end{abstract}

Correspondence to: Toshimasa Yamazaki, t-ymzk@bio.kyutech.ac.jp

Keywords: Electroencephalography, Network, Dementia, Synchronization Likelihood, Leave-One-Out Cross Validation, Euclidean Distance

Received: June 11, 2020 Accepted: July 27, $2020 \quad$ Published: July 30, 2020

Copyright (c) 2020 by author(s) and Scientific Research Publishing Inc.

This work is licensed under the Creative Commons Attribution International License (CC BY 4.0).

http://creativecommons.org/licenses/by/4.0/

\section{(c) (i) Open Access}

\section{ABSTRACT}

Objective: To establish a practical method for discriminating dementia groups and healthy elderlies, by using scalp-recorded electroencephalograms (EEGs). Methods: 16-ch EEGs were recorded during resting state for 39 dementia groups and 11 healthy elderlies. The connectivity between any two electrodes was estimated by synchronization likelihood (SL). The brain networks were constructed by normalized SL values. The present leave-one-out cross validation (LOOCV) required the Euclidean distance between any two subjects having 120-dimensional vectors concerned with the SL values for six frequency bands. In order to investigate factors which would affect the LOOCV results, principal component analysis (PCA) was applied to all the subjects. Results. The accuracy for the upper alpha yielded more than $80 \%$ and $70 \%$ in the dementia groups and the healthy elderlies, respectively. The LOOCV result could be explained in terms of brain networks such as executive control network (ECN) and default mode network (DMN) characterized by factor loadings of principal components. Conclusions: Dementia groups and healthy elderlies could be characterized by principal components of SL values between all the electrode pairs, even less connections, which revealed disruption and preservation of DMN and ECN. Significance: This study will provide a simple and practical method for discriminating dementia groups from healthy elderlies by scalp-recorded EEGs. 


\section{INTRODUCTION}

Alzheimer's disease $(\mathrm{AD})$ is the most common form of dementia in the world. 50 million people are suffering from dementia and over 60 percent of dementia cases are Alzheimer's disease. In addition, these patients are likely to reach about 152 million people by 2050 [1]. AD is an irreversible neurodegenerative disease characterized by progressive impairment of memory, and loss of cognitive functions that affect a person's ability to perform daily activities. The evolution of the disease frequently follows some stages and symptoms gradually worsen over a number of years [2].

One of the difficult problems of $\mathrm{AD}$ is diagnosis. As things stand at the moment, there is no single test way for AD. Physicians are using several methods and tools to make a diagnosis, which included medical and family history, cognitive tests and physical and neurologic examinations, and brain imaging [3]. Also, several days or weeks are needed to complete the required tests and for the physician to interpret the results and make a diagnosis [3]. Therefore, AD diagnosis using easy and inexpensive techniques will provide better care to patients.

Current theories of $\mathrm{AD}$ postulate that in the brain networks the decline arises from alterations in functional integration of distributed brain systems or from structural disconnection between regions because of white matter damage. Such brain networks during resting states have been known as executive control network (ECN) [4], default mode network (DMN) [5] and salience network (SN) [6]. The ECN is a brain network responsible for high-level cognitive functions, notably the control of attention and working memory. The DMN is a large-scale network of brain areas that form an integrated system for self-related cognitive activity, including autobiographical, self-monitoring and social functions and the SN is a brain network involved in the orientation of attention to the most homeostatically relevant (salient) of ongoing intrapersonal and extrapersonal events [7].

The previous functional magnetic resonance imaging (fMRI) studies have reported disrupted connectivity within the DMN $[8,9]$, the SN $[9,10]$, the ECN $[9,11]$ and the SN-ECN disconnection [12] in AD patients. However, there have been a few approaches using electroencephalography (EEG) [13-16], which is one of easier and cheaper methods, and magnetoencephalography (MEG) [17].

From the point of view of cognitive functions and tests, Alzheimer's dementia has been known to be classified into the following five stages at least: preclinical stage, the second stage (Mild Cognitive Impairment (MCI)), mild stage (MMSE (Mini Mental Status Examination) > 20), moderate stage (MMSE between 10 and 20) and severe stage [2]. In particular, an early diagnosis of Alzheimer's disease in MCI and mild AD stages has become very important.

In this study, we will find out scalp-recorded-EEG-based brain networks, by which dementia groups with MCI and mild, moderate and severe AD stages are compared with healthy elderlies. The brain networks are constructed from 16-ch EEGs in six frequency bands (delta $(0-4 \mathrm{~Hz})$, theta $(4-8 \mathrm{~Hz})$, lower alpha $(8-10 \mathrm{~Hz})$, upper alpha $(10-13 \mathrm{~Hz})$, beta $(13-30 \mathrm{~Hz})$, gamma $(30-45 \mathrm{~Hz}))$. Graphical representations of the brain networks based on graph theory are formed by a functional connectivity measure called synchronization likelihood (SL) [18] which is a generalized synchronization measure between any two electrodes. Then, each subject is characterized by an average of the SL values across all the electrode pairs. Next, in order to examine the possibility of AD diagnosis, we will try leave-one-out cross validation (LOOCV). Finally, we will discuss the LOOCV results in terms of existing brain networks such as ECN, DMN and SN.

\section{MATERIALS AND METHODS}

\subsection{EEG Measurement}

The present subjects consist of 39 dementias and 11 healthy elderlies, Table 1 shows those subjects information. EEG measurements were performed at Teikyo University School of Medicine, through a Nihon Kohden EEG-1224 for the subjects. The device was equipped with $16 \mathrm{Ag} / \mathrm{AgCl}$ electrodes (a Nihon Kohden H503A) were attached at Fp1, Fp2, F3, F4, C3, C4, P3, P4, O1, O2, F7, F8, T3, T4, T5, and T6 on 
the basis of the International 10 - 20 System (Figure 1). The sampling rate was set to be $500 \mathrm{~Hz}$. The filter setting was high pass filter $(=120 \mathrm{~Hz})$. All the subjects were instructed to lie on their back in the resting state with their closed eyes for 5 minutes at least. The study protocol illustrated in Figure 2, including all EEG data analyses, was approved by The Ethics Committee for Human Subject Researches, Faculty of Computer Science and Systems Engineering, Kyushu Institute of Technology. All EEG data was checked in the EEG lab (provided by Swartz Center for Computational Neuroscience) and deleted at least 1 minute from the starting time and the EEG data after 1 minute were used for the data analyses as use stable EEG data.

All the EEG data was passed through band pass filter that separated the EEG data into six frequency bands: delta $(0-4 \mathrm{~Hz})$, theta $(4-8 \mathrm{~Hz})$, lower alpha $(8-10 \mathrm{~Hz})$, upper alpha $(10-13 \mathrm{~Hz})$, beta $(13-30$ $\mathrm{Hz}$ ), gamma $(30-45 \mathrm{~Hz})$ (Figure 2).

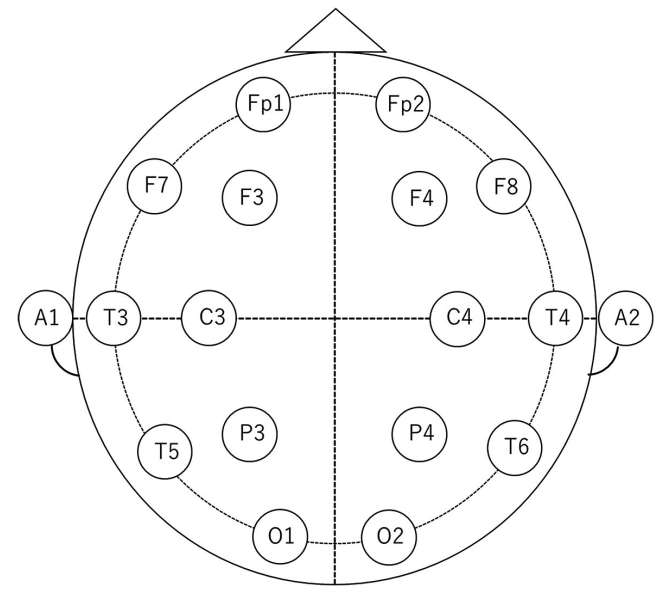

Figure 1. Electrode positions.

(1) Recording EEG in resting state (2) Filtering in six frequency bands

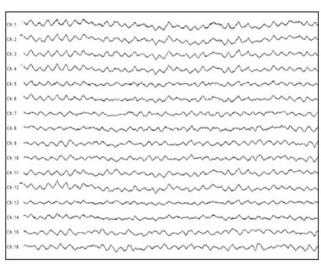

(5) Divided community from non-weighted matrix

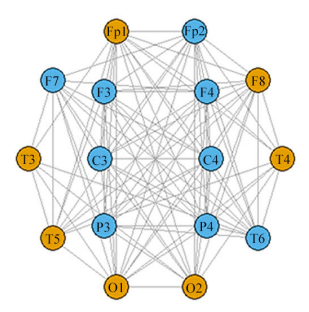

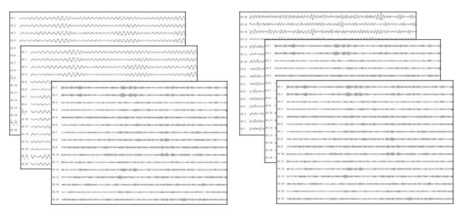

(6) Leave one out cross validation
( 3 ) Calculating the SL and (4) Constructing network from

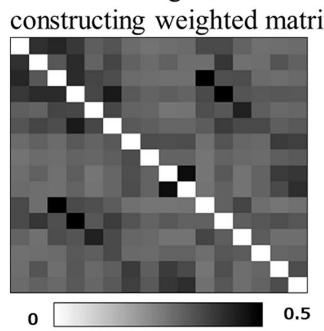

weighted matrix

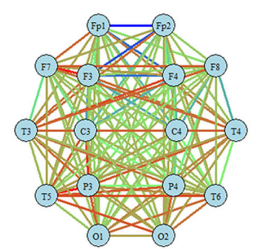

(7) Principal component analysis in upper alpha band
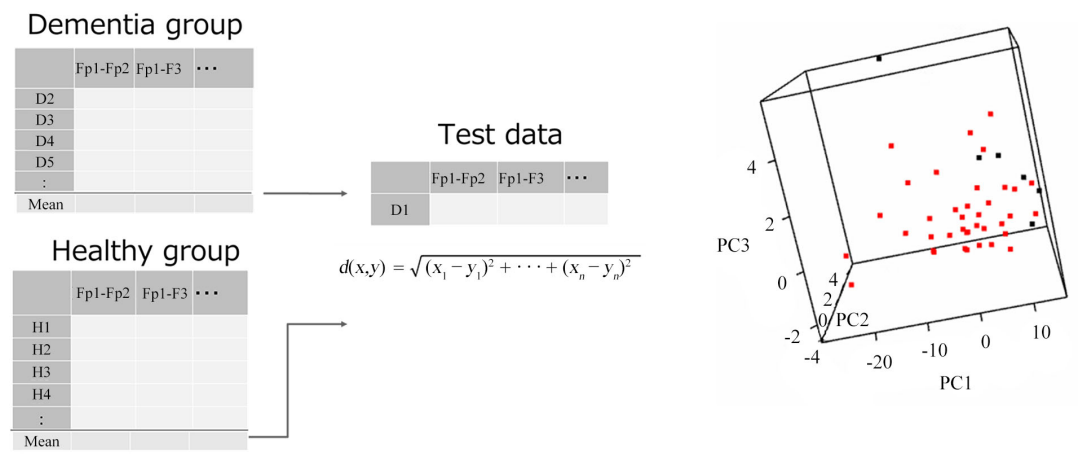

Figure 2. Flow of from EEG recording to data analysis. 
Table 1. Subject information.

\begin{tabular}{ccc}
\hline & Dementia groups & Healthy elderlies \\
\hline $\mathrm{N}^{*}$ & $39^{* * *}$ & 11 \\
Age $($ mean $)$ & $71-96(85.28)$ & $67-89(81.36)$ \\
Sex $F / \mathrm{M}^{* *}$ & $17 / 22$ & $3 / 8$ \\
\hline
\end{tabular}

${ }^{\star}$ The number of subjects, ${ }^{* *} \mathrm{~F}=$ female, $\mathrm{M}=$ male, ${ }^{* *}: 23(10 \leq \mathrm{MMSE} \leq 20) ; 3$ (MMSE $\left.>20\right) ; 13$ (MCI).

\subsection{Synchronization Likelihood (SL)}

After the EEG measurement, SL was calculated between any two electrodes. The SL is a measure of synchronization between two times series that is sensitive to linear and non-linear independencies. The basic principle of the SL is to divide each time series into a series of "pattern" (roughly, brief pieces of time series containing a few cycles of the dominant frequency) and to search for a recurrence of these patterns. Figure 3 [19] illustrated the state vectors and synchronization likelihood parameters $(L, m, W 1, W 2$ and $s)$ with respect to the time series of channels A and B. Figure 4 [19] shows the SL between two channels in terms of state vectors and critical distances. The reference vector of channel $\mathrm{A}$ is denoted $X_{\mathrm{A}, \mathrm{i}}$ (thick line square) here chosen to have embedding dimension $m=3$ samples (small ticks) and lag $L=2$ samples (dots). The reference vector is compared with state vectors (squares) $X_{\mathrm{A}, \mathrm{j}}(j= \pm 1,2 \ldots n)$ within a window of $W 2$. State vectors starting at times $\mathrm{j}$ in the time interval outside the window $W 1$ and within the window $W 2$ (windows centered at time $i$ ) are compared with the reference vector. The time series is indicated with a solid horizontal line and the time intervals are indicated with a dashed line. The vectors $X_{\mathrm{A}, \mathrm{j}}$, which are closer to the reference vector $X_{\mathrm{A}, \mathrm{i}}$ than the critical distance, $r_{\mathrm{A}}$ (Figure 4) are represented in white. Whereas the vectors that are not within the critical distance are represented in grey, and termed recurrences are represented with white squares. Similarly to channel A, a reference vector $X_{\mathrm{B}, \mathrm{i}}$ is compared with all state vectors $X_{\mathrm{B}, \mathrm{j}}(j=1,2 \ldots n)$. If the vectors are closer to $X_{\mathrm{B}, \mathrm{i}}$ than $r_{\mathrm{B}}$ then they are represented in white, otherwise in grey. The SL value is the number of simultaneous recurrences in channels A and B divided by the total number of recurrences within channels. In this situation, the SL value is 0.5 . These procedures which convert the SL values between the 16 electrodes into the adjacency matrix are illustrated in Figure 2 . The Lab_SL2 which is the Matlab script for calculating the SL was used in this study.

\subsection{Brain Network Construction}

SL values show strength of connectivity of each of the two electrodes (Figure 4) [19]. For each electrode pair, averages of SL values in the healthy controls were calculated, and then SL values in the AD patients were divided by the healthy averages. Thus, from the brain network of the healthy group, that of the AD group was constructed, as shown in Figure 5.

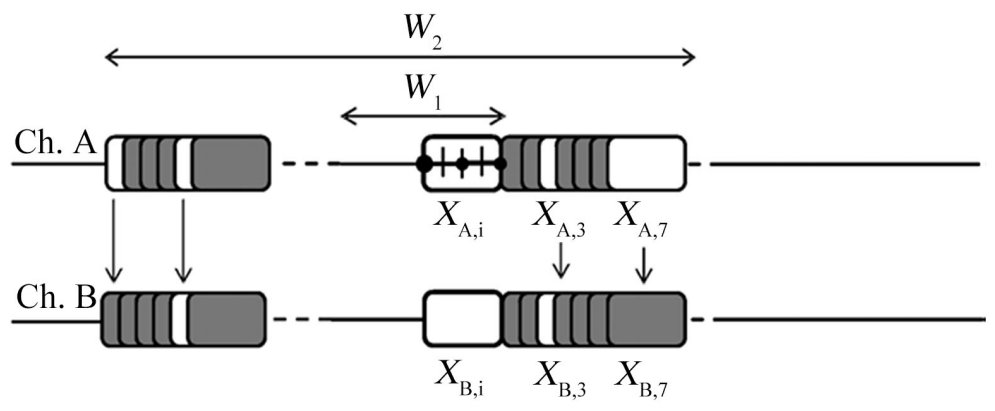

Figure 3. State vectors describing two time series in channels $\mathrm{A}$ and $\mathrm{B}\left(X_{\mathrm{A}, \mathrm{i}}, X_{\mathrm{B}, \mathrm{i}}\right)$, and pameters for calculating SL $\left(W_{1}, W_{2}\right)$. 


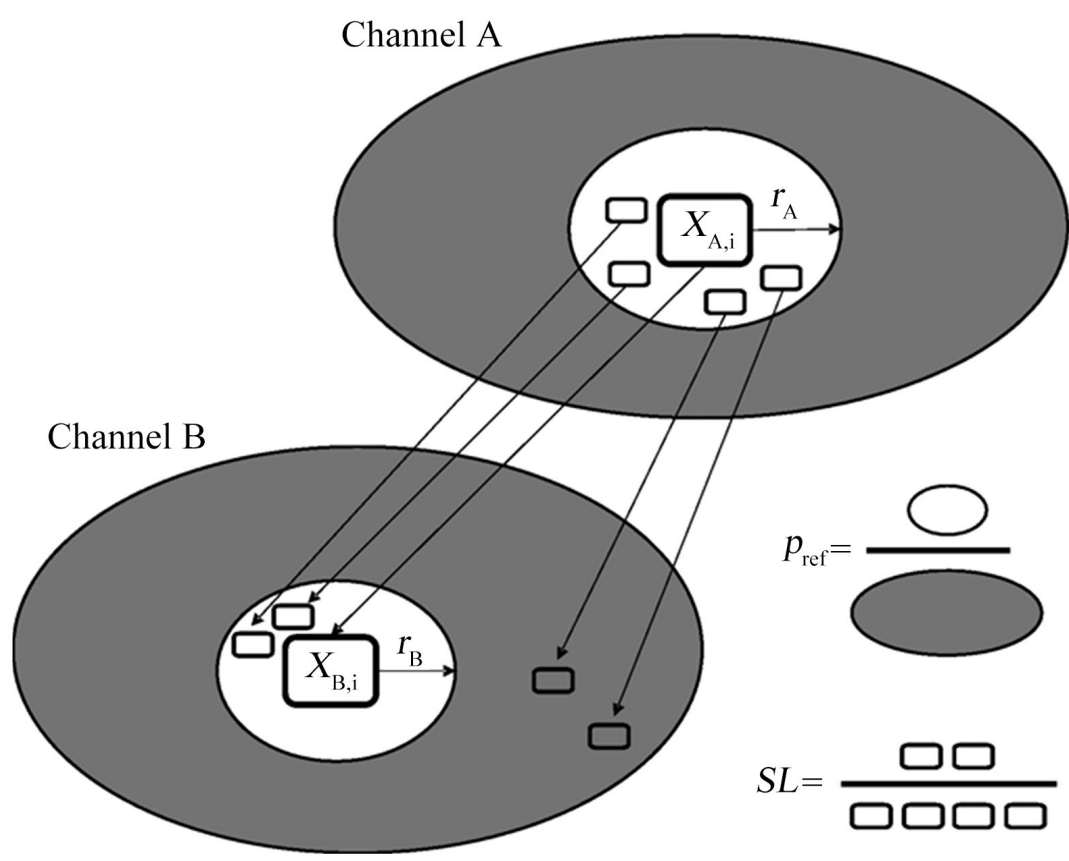

Figure 4. Representation of SL between two channels A and B by state vectors and critical distances $\left(r_{\mathrm{A}}, r_{\mathrm{B}}\right)$.
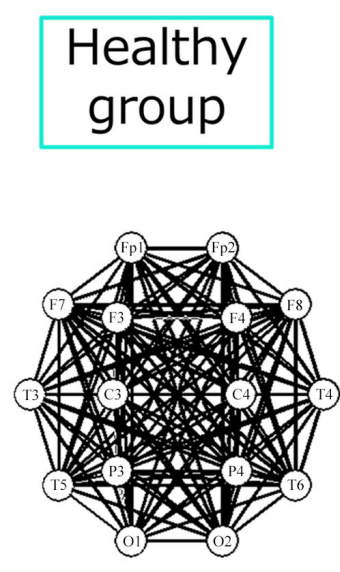

Set the SL value of
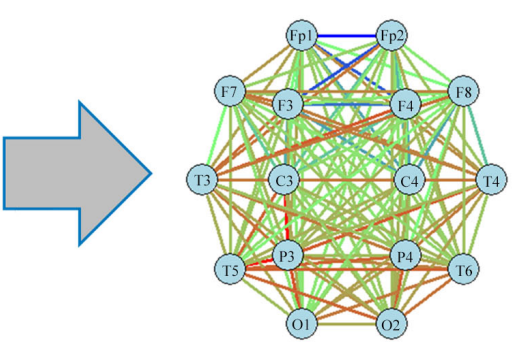

the healthy SL value=1
High
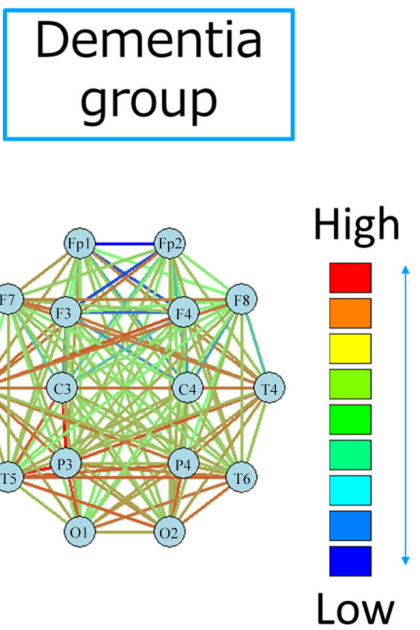

Figure 5. Construction of AD network by normalized SL values.

\subsection{Brain Network Modularization}

The graph structure of the brain network was represented by the nodes and edges that correspond to the electrode positions and the SL values, respectively, where the present network was undirected and unweighted (binary) in this research (Figure 5). The brain network was divided into some community groups, which could predict any cognitive function. The network constructed by SL average values was divided into two graphs, so that one is lower than the healthy group and other higher than the healthy group (Figure 6). Moreover, the optimal community structure of a graph was found by maximizing the modularity measure over all possible partitions [20]. 

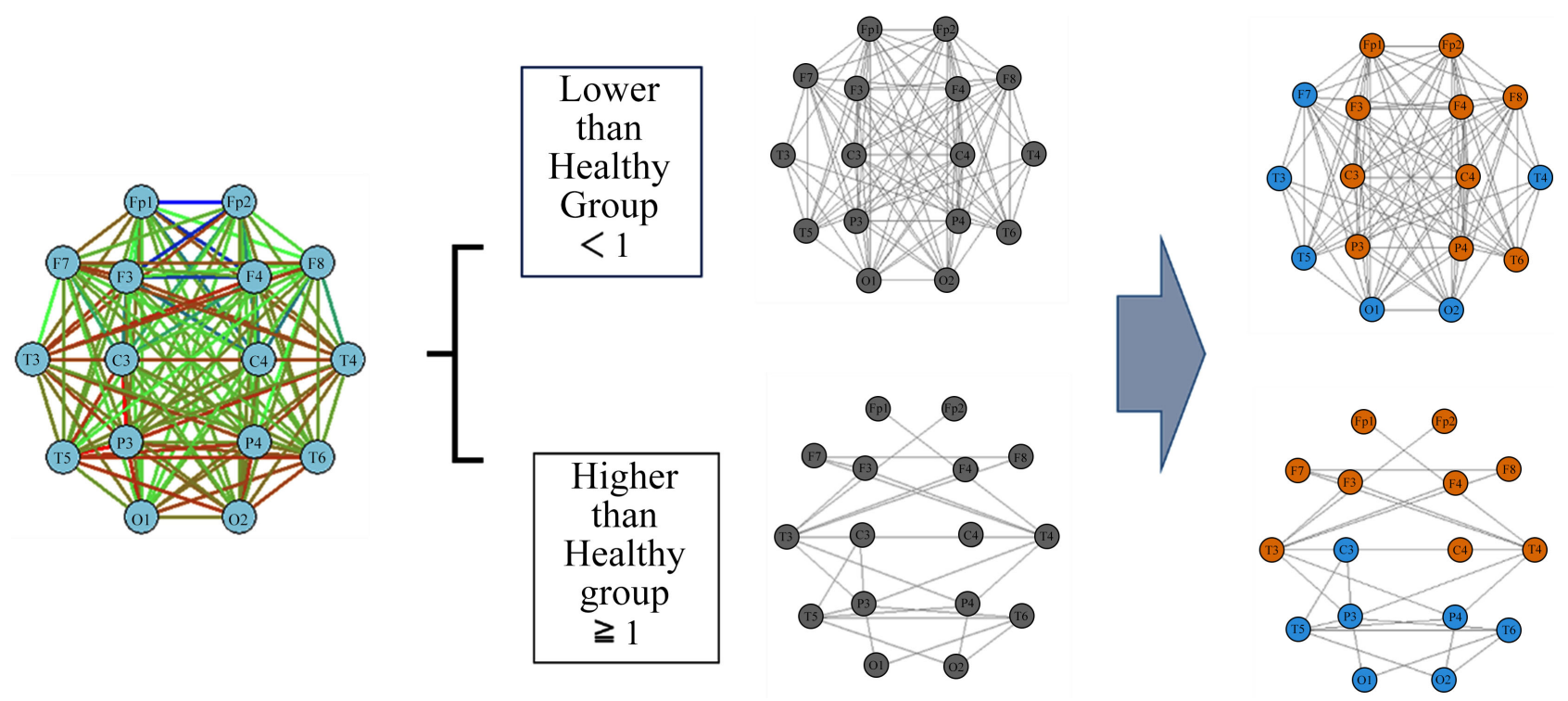

Figure 6. Modularization of brain network using graph theory.

\subsection{Leave-One-Out Cross Validation (LOOCV)}

LOOCV uses a single test data from the original samples as the validation data, and the remaining data as the training data (Figure 2). Firstly, we picked one test data, and calculated "AD and healthy averages" for the remaining data. The "AD and healthy averages" are defined to be averages across subjects in each group for each electrode pair.

For one test data, $120(=16 \times 15 / 2)$-dimensional Euclidean distance between the subject and "the dementia or healthy average" was calculated. If the Euclidean distance in the dementia group is smaller than that in the healthy group, the test data is determined to be from "dementia", otherwise from "healthy". This procedure was repeated for all the test data.

\subsection{Principal Component Analysis (PCA)}

In order to investigate factors (brain networks) which could affect the above LOOCV results, principal component analysis (PCA) is applied to all the subjects in terms of all the electrode pairs (Figure 2).

\section{RESULTS}

\subsection{Brain Network Construction}

Figure 7 shows the dementia group networks for each frequency band compared with the healthy elderly controls. The SL values are averaged for all the time-series data in the dementia group and the healthy elderly group.

Figure 7 also visualizes whether the SL average value between the nodes is higher or lower than the healthy control value. In all the frequency bands except for delta, the connections between Fp1-Fp2 in the dementia group are weaker than the elderly control group. In theta and gamma bands, the connectivity of the dementia groups is lower than that of the elderly controls.

From non-overlapping community detection, nodes consisting of the same community could have high functional relevance. Figure 8 and Figure 9 show that the alpha band network of dementia groups is distributed in the whole of the brain, indicating that SL values in dementia group are higher than those in elderly controls. Moreover, the gamma band network of dementia groups is separated between the left and right sides of the brain. In the theta band, the connectivity in the dementia groups is almost completely lower than that in the elderly controls. 


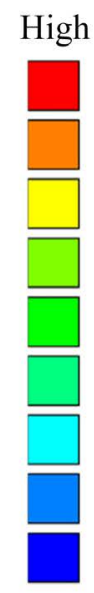

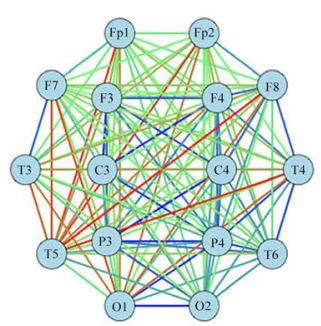

Delta

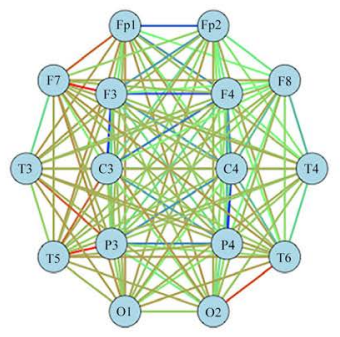

Upper alpha

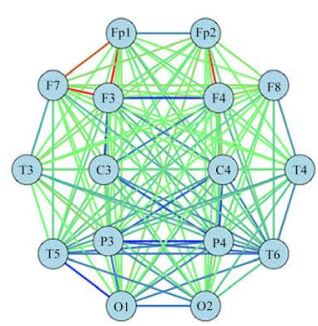

Theta

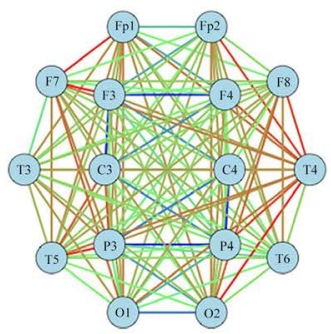

Beta

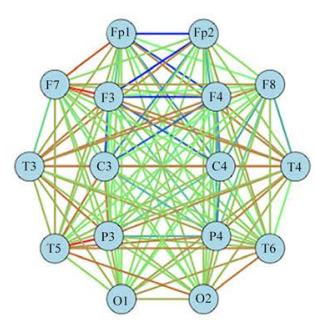

Lower alpha

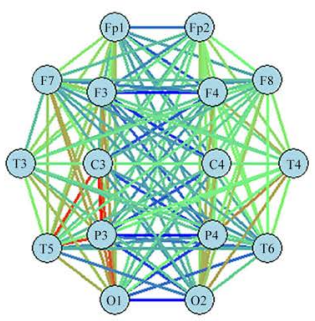

Gamma

Figure 7. Brain networks of dementia groups for six frequency bands after the normalization where all the SL values for the healthy controls are assumed to be one.

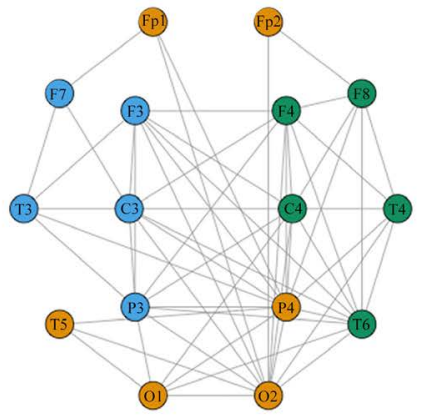

Delta

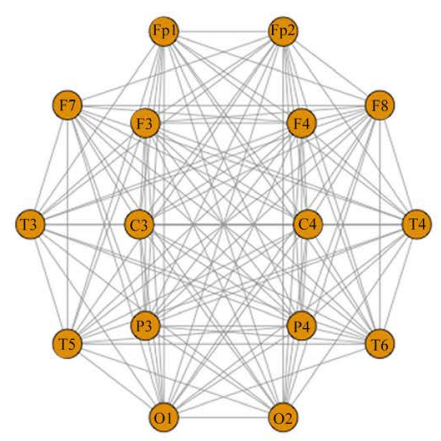

Upper alpha

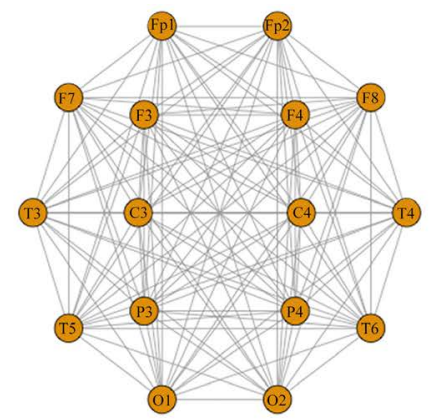

Theta

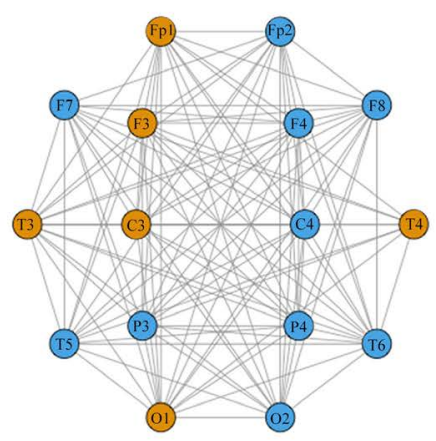

Beta

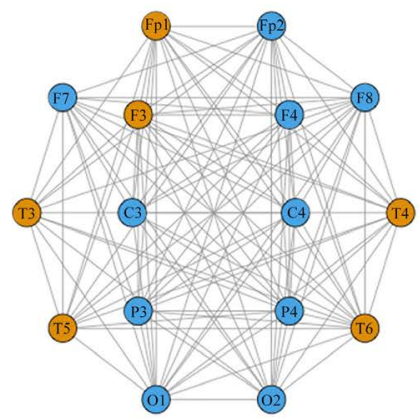

Lower alpha

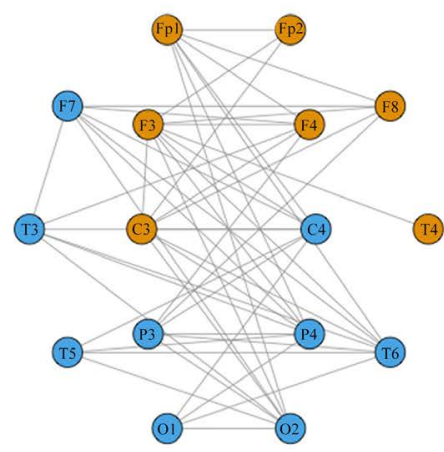

Gamma

Figure 8. Non-overlapping community when elderly controls $>$ dementia groups. 


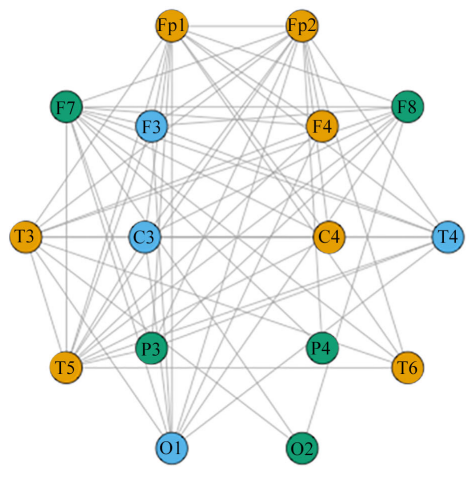

Delta

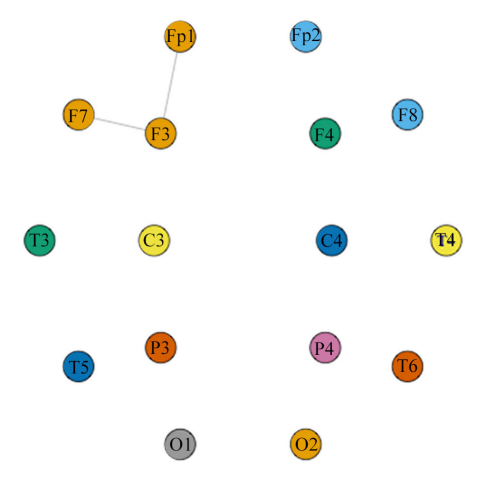

Theta

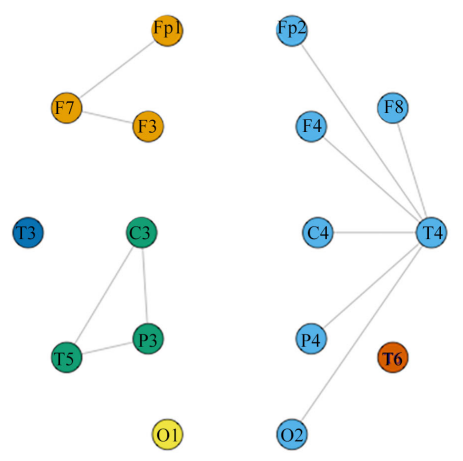

Beta

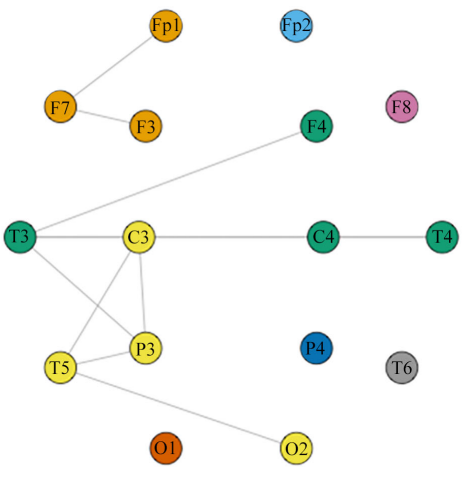

Lower alpha

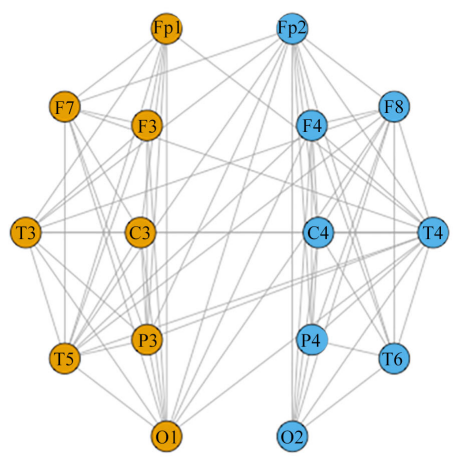

Gamma

Figure 9. Non-overlapping community when dementia groups $>$ elderly controls.

\subsection{LOOCV}

Table 2 shows the accuracy rate for "dementia" and "healthy". In the dementia group, three bands (lower alpha, upper alpha and beta) show the high accuracy, and also in the healthy group, two bands (theta and upper alpha) also show the high rate. So, the upper alpha band was applied to PCA.

Figure 10 shows plots of all the subjects by the first two principal components (PC1 and PC2) in the upper alpha band. Table 3 depicts the cumulative proportion of the PCs. It follows that PC1 and PC2 explain only $56 \%$ of the SL value variation. In Figure 10, orange dots represent healthy controls, and blue dots represent dementia groups. This figure shows that the dementia groups and healthy groups might be separated (as mentioned in Discussion). To find which components affected the PCA results, factor loadings were calculated for all the electrode pairs (Figure 11). Figure 11 also depicts three lines indicating main axes of factor loading variation in PC1 and PC2 by inspection. These lines are represented by PC1 factor loading values with larger than 0.85 , PC2 factor loading values with larger than 0.4 and those with less than -0.4 . And, Figures 12-14 show brain networks which were characterized by the three constraints.

\section{DISCUSSION}

The frontal cortex helps mediate the working memory that is used for temporary storage and manipulation of information and involved in many higher cognitive functions [21]. Therefore, well-known symptoms of $\mathrm{AD}$ are related with functions of the frontal cortex [22]. Because our results (Figure 7) indicated low connectivity in the frontal cortex, the brain networks might reveal dysfunction of the frontal cortex. Figure 8 and Figure 9 suggested that the networks of the AD group might have some characteristics so that the network of $\mathrm{AD}$ patients could distinguish from that of the healthy elderly controls. 


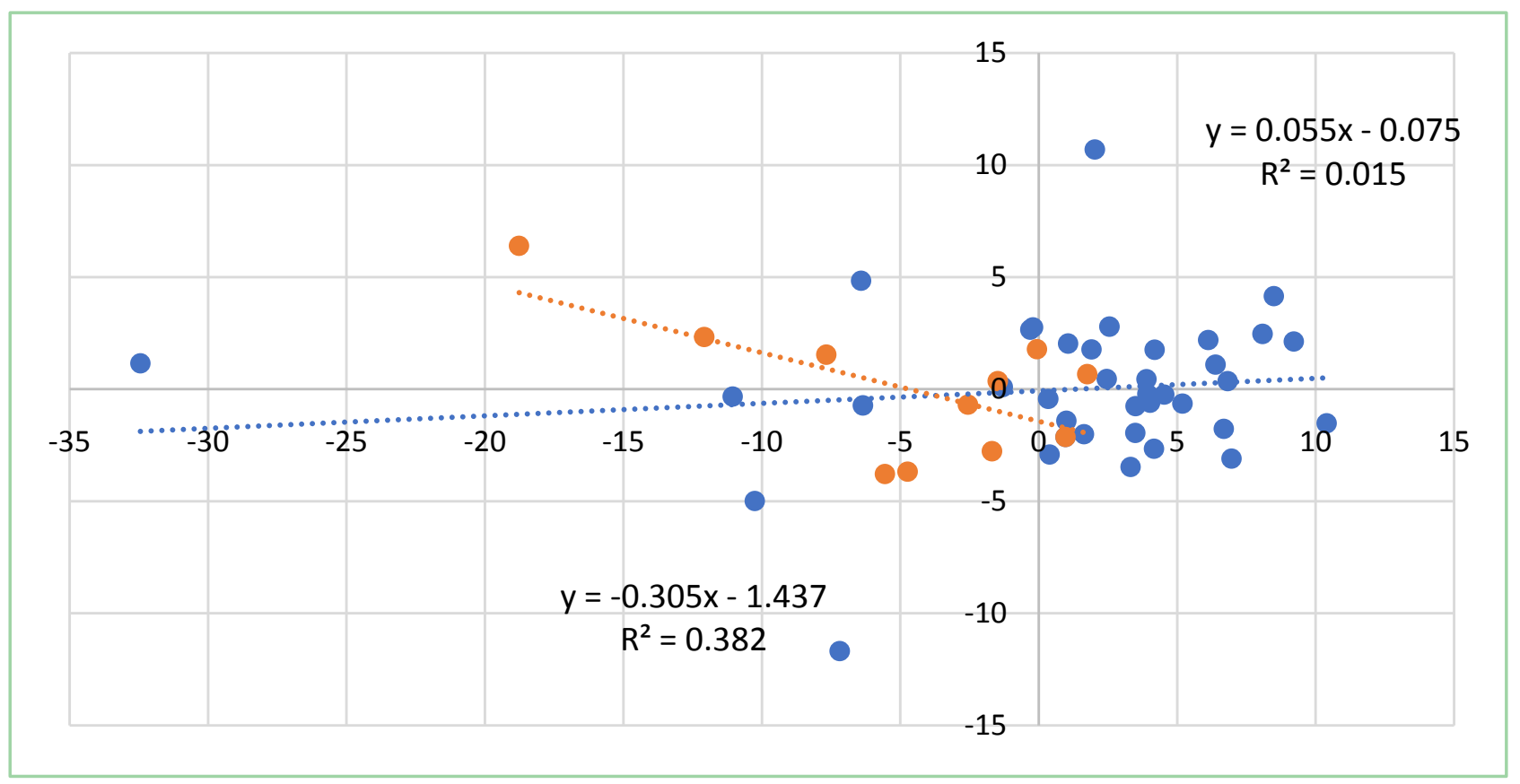

Figure 10. Plots of all the subjects in terms of the first two principal components.

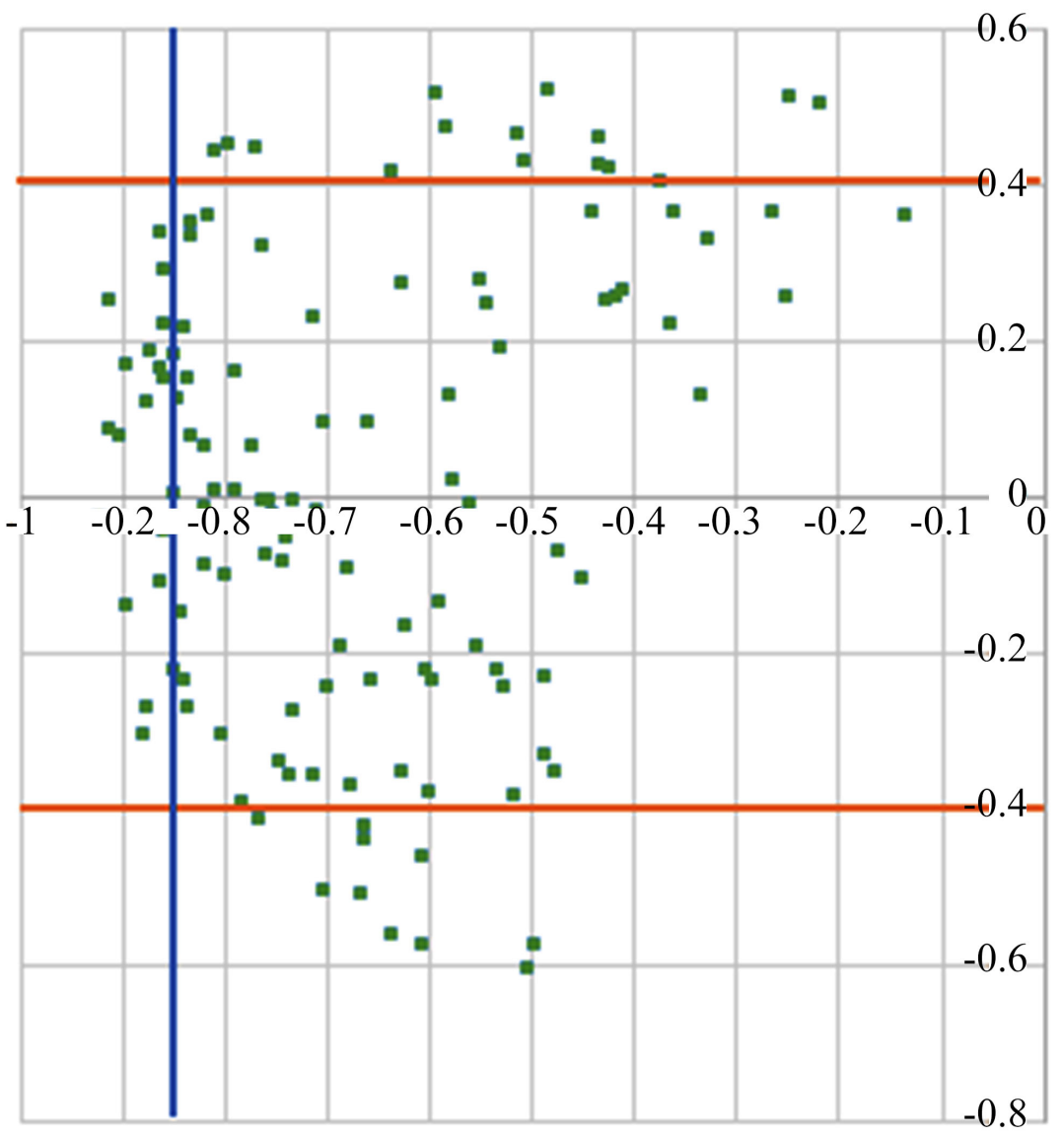

Figure 11. Plots of all the electrode pairs in terms of factor loadings of PC1 and PC2. 


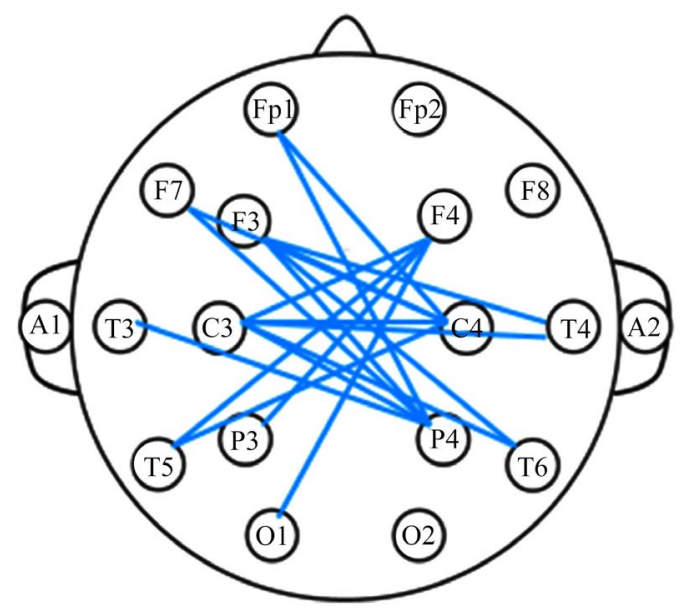

Figure 12. Brain network with PC1 factor loadings of larger than 0.85 .

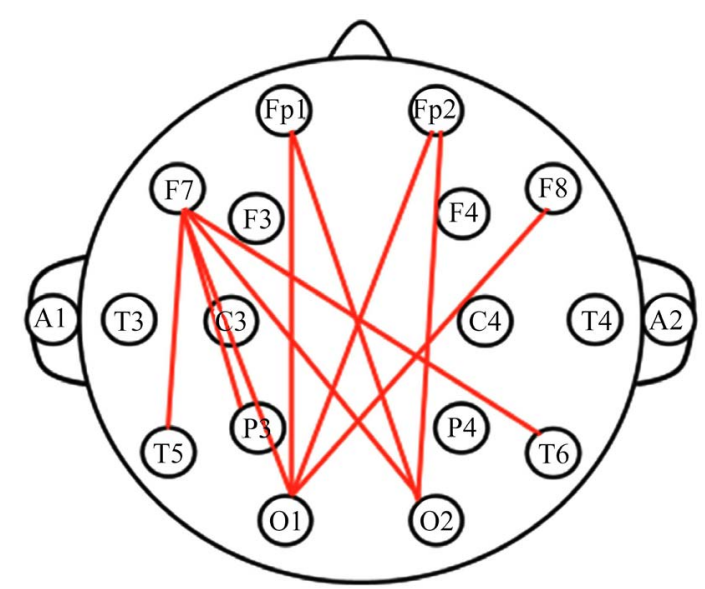

Figure 13. Brain network with PC2 factor loadings of less than -0.4 .

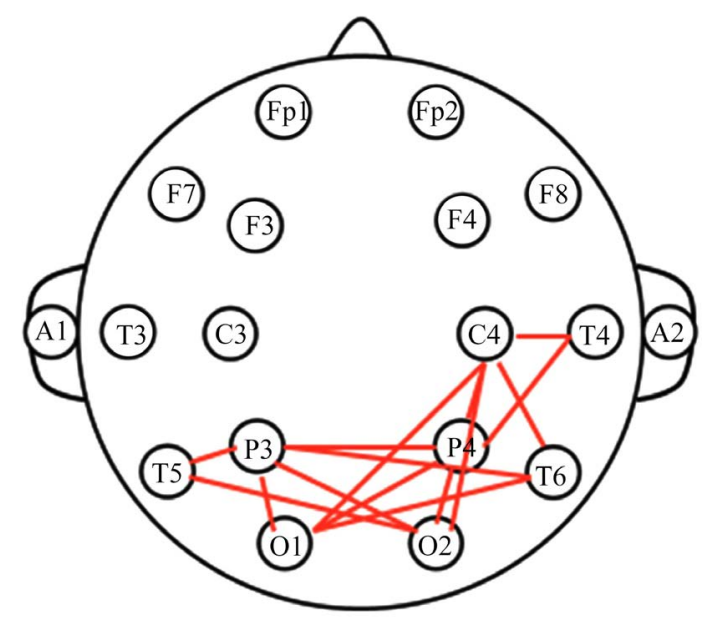

Figure 14. Brain network with PC2 factor loadings of larger than 0.4 . 
Table 2. Results on LOOCV using the Euclidean distances in six frequency bands.

\begin{tabular}{ccccccc}
\hline & delta & theta & lower $\boldsymbol{a}$ & upper $\boldsymbol{\alpha}$ & beta & gamma \\
\hline Dementia group & $72 \%$ & $64 \%$ & $82 \%$ & $82 \%$ & $82 \%$ & $51 \%$ \\
Healthy & $64 \%$ & $82 \%$ & $36 \%$ & $73 \%$ & $64 \%$ & $91 \%$ \\
\hline
\end{tabular}

Table 3. Cumulative proportion of the upper alpha band.

\begin{tabular}{cccccc}
\hline & PC1 & PC2 & PC3 & PC4 & PC5 \\
\hline Standard deviation & 7.5527 & 3.25905 & 3.10177 & 2.74594 & 2.23793 \\
Proportion of variance & 0.4754 & 0.08851 & 0.08018 & 0.06283 & 0.04174 \\
Cumulative proportion & 0.4754 & 0.56387 & 0.64404 & 0.70688 & 0.74861 \\
\hline
\end{tabular}

The present LOOCV result yielded over $80 \%$ accuracy in the upper alpha band, which is the same as accuracy in classification tasks comparing AD patients and aged-matched healthy controls [23-28]. In order to investigate factors which could affect the present accuracy, PCA was applied to SL values in terms of any two electrodes. Figure 10 characterized each subject in terms of the first two principal components. Visual inspection of this figure roughly suggests that the dementia groups could be characterized only by the PC1 and the healthy controls by a combination of the PC1 and the PC2. Actually, the coefficients of correlation were 0.1233 and -0.6188 for the dementia groups and the healthy controls, respectively (Figure 10). Moreover, Figures 12-14 show brain networks determined by factor loadings of the two PCs (PC1 and PC2), which were defined to be the PC1 factor loadings of more than 0.85 , the PC2 factor loading of less than -0.4 and the PC2 factor loading of larger than 0.4, respectively, with reference to Figure 11.

Generally, brain networks during resting state can be defined on the basis of structural connectivity or functional interdependence from some large scale ones such as DMN, ECN and SN. The ECN is characterized by the dorsal prefronto-parietal network mainly involving the lateral prefrontal cortex (LPC) and the posterior parietal cortex (PPC) [21, 29]. The DMN contains the medial prefrontal cortex (MPC), the posterior cingulate cortex (PCC), the inferior parietal lobe (IPL), the lateral temporal cortex (LTC) and the hippocampal formation (HF) [30], each of which organizes a different functional area. The two networks during resting states do not always conflict, sometimes cooperate between them [31] and affect each other. In particular, it has been reported that the synchronization of the fast alpha reflects the DMN function and is responsible for top-down processing [32].

Figure 12 seems to reveal the connection of DMN. The PCC, an occipital-core area of DMN, corresponds to the P3-P4-T5-T6, and the MPFC to the Fp1-Fp2-F3-F4 in the present experiment. Because, in this study, the EEGs were recorded during resting state with closed eyes, the brain networks such as DMN and ECN are likely to be formed.

In contrast, Figure 13 seems to reveal the brain network with the connection of ECN according to the PC2 factor loadings. The DMN is predominant in the absence of any external tasks, while the ECN is a resting-state-task-related network [7,33-37]. Moreover, it has been well known that the DMN and the ECN have negative correlation (e. g., [36, 38-44]). This could be a reason why it is difficult to divide into dementia group and healthy one in the PC2. Additionally, Figure 14 shows occipitally dominant networks in the upper alpha band, which is supported by alpha activity in the occipital cortex (e.g., [45]).

Finally, dementia groups and healthy elderlies could be characterized by principal components of SL values between all the electrode pairs, revealing disruption and preservation of DMN and ECN. LOOCV using much less between-electrode connections yielded the same accuracy (85\% and 73\% for dementia groups and healthy elderlies, respectively) as Table 1 . This reduction was executed by a model by AIC (Akaike information criterion) in a stepwise algorithm (e.g., [46]). The resultant reduction is shown in Figure 15. Thus, this study will provide an easy and practical method for discriminating dementia groups from healthy elderlies by scalp-recorded EEGs. 


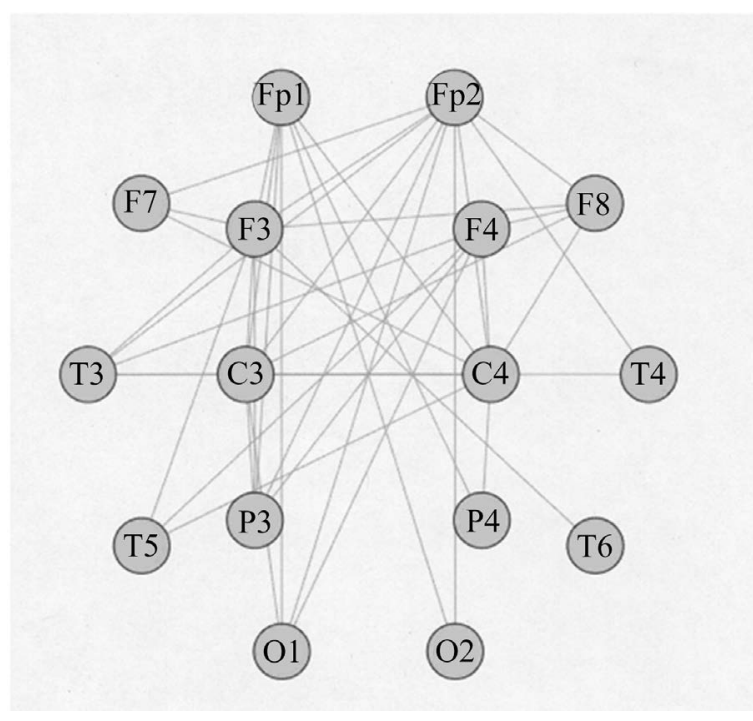

\section{Figure 15. Distribution of between-electrode connections reduced by a model by AIC in a stepwise algorithm.}

\section{CONCLUSION}

We obtained brain functional connectivity networks (BFCNs) for dementia groups including $\mathrm{AD}$ and MCI and elderly controls using scalp-recorded EEGs. The BFCNs were constructed from connectivity strength described by SL values at each electrode pair. Principal components of the SL values characterized the subjects in terms of disruption and preservation of default mode network (DMN) and executive control network (ECN) for the dementia groups and the elderly controls, respectively. Thus, this study will provide an easy and practical method for discriminating dementia groups from healthy elderlies using scalp-recorded EEGs.

\section{CONFLICTS OF INTEREST}

The authors declare no conflicts of interest regarding the publication of this paper.

\section{REFERENCES}

1. Alzheimer's Disease International (2018) World Alzheimer Report 2018. The State of the Art of Dementia Research: New Frontiers. Alzheimer's Disease International, London.

2. Houmani, N., Vialatte, F., Gallego-Jutglà, E., Dreyfus, G., Nguyen-Michel, V.-H., Mariani, J. and Kinugawa, K. (2018) Diagnosis of Alzheimer's Disease with Electroencephalography in a Differential Framework. PLoS ONE, 13, e0193607. https://doi.org/10.1371/journal.pone.0193607

3. Alzheimer's Association (2018) Alzheimer's Association Report 2018, 2018 Alzheimer's Disease, Facts and Figures. Alzheimer's Association, Chicago.

4. Corbetta, M. and Shulman, G.L. (2002) Control of Goal-Directed and Stimulus Driven Attention in the Brain. Nature Review Neuroscience, 3, 201-215. https://doi.org/10.1038/nrn755

5. Raichle, M.E., MacLeod, A.M., Snyder, A.Z., Powers, W.J., Gusnard, D.A. and Shulman, G.L. (2001) A Default Mode of Brain Function. Proceedings of the National Academy Sciences, 98, 676-682.

https://doi.org/10.1073/pnas.98.2.676

6. Seeley, W.W., Menon, V., Schatzberg, A.F., Keller, J., Glover, G.H., Kenna, H., Reiss, A.L. and Greicius, M.D. (2007) Dissociable Intrinsic Connectivity Networks for Salience Processing and Executive Control. Journal of 
Neuroscience, 27, 2349-2356. https://doi.org/10.1523/JNEUROSCI.5587-06.2007

7. Bressler, S.L. and Menon, V. (2010) Large-Scale Brain Networks in Cognition: Emerging Methods and Principles. Trends in Cognitive Sciences, 14, 277-290. https://doi.org/10.1016/j.tics.2010.04.004

8. Greicius, M.D. and Menon, V. (2004) Default-Mode Activity during a Passive Sensory Task: Uncoupled from Deactivation But Impacting Activation. Journal of Cognitive Neuroscience, 16, 1484-1492. https://doi.org/10.1162/0898929042568532

9. Brier, M.R., Thomas, J.B., Snyder, A.Z., Benzinger, T.L., Zhang, D., Raichle, M.E., Holtzman, D.M., Morris, J.C. and Ances, B.M. (2012) Loss of Intranetwork and Internetwork Resting State Functional Connections with Alzheimer's Disease Progression. Journal of Neuroscience, 32, 8890-8899.

https://doi.org/10.1523/JNEUROSCI.5698-11.2012

10. Chen, G., Zhang, H.Y., Xie, C., Chen, G., Zhang, Z.J., Teng, G.J. and Li, S.J. (2013) Modular Reorganization of Brain Resting State Networks and Its Independent Validation in Alzheimer's Disease Patients. Frontiers in $\mathrm{Hu}$ man Neuroscience, 7, Article 456. https://doi.org/10.3389/fnhum.2013.00456

11. Li, R., Wu, X., Fleisher, A.S., Reiman, E.M., Chen, K. and Yao, L. (2012) Attention-Related Networks in Alzheimer's Disease: A Resting Functional MRI Study. Human Brain Mapping, 33, 1076-1088.

https://doi.org/10.1002/hbm.21269

12. Dai, Z., Yan, C., Li, K., Wang, Z., Wang, J., Cao, M., Lin, Q., Shu, N., Xia, M., Bi, Y. and He, Y. (2015) Identifying and Mapping Connectivity Patterns of Brain Network Hubs in Alzheimer’s Disease. Cerebral Cortex, 25, 3723-3742. https://doi.org/10.1093/cercor/bhu246

13. Petrosian, A.A., Prokhorov, D.V., Lajara-Nanson, W. and Schiffer, R.B. (2001) Recurrent Neural Network-Based Approach for Early Recognition of Alzheimer's Disease in EEG. Clinical Neurophysiology, 112, 1378-1387. https://doi.org/10.1016/S1388-2457(01)00579-X

14. Stam, C.J., Montez, T., Jones, B.F., Rombouts, S.A., van der Made, Y., Pijnenburg, Y.A. and Scheltens, P. (2005) Disturbed Fluctuations of Resting State EEG Synchronization in Alzheimer's Disease. Clinical Neurophysiology, 116, 708-715. https://doi.org/10.1016/j.clinph.2004.09.022

15. Hsiao F.-J., Wang, Y.-J., Yan, S.-H., Chen, W.-T. and Lin, Y.-Y. (2013) Altered Oscillation and Synchronization of Default-Mode Network Activity in Mild Alzheimer's Disease Compared to Mild Cognitive Impairment: An Electrophysiological Study. PLoS ONE, 8, e68792. https://doi.org/10.1371/journal.pone.0068792

16. Vecchio, F., Miraglia, F., Piludu, F., Granata, G., Romanello, R., Caulo, M., Onofrj, V., Bramanti, P., Colosimo, C. and Rossini, P.M. (2017) "Small World" Architecture in Brain Connectivity and Hippocampal Volume in Alzheimer's Disease: A Study via Graph Theory from EEG Data. Brain Imaging and Behavior, 11, 473-485. https://doi.org/10.1007/s11682-016-9528-3

17. de Haan, W., van der Flier, W.M., Koene, T., Smits, L.L., Scheltens, P. and Stam, C.J. (2012) Disrupted Modular Brain Dynamics Reflect Cognitive Dysfunction in Alzheimer's Disease. Neuroimage, 59, 3085-3093. https://doi.org/10.1016/j.neuroimage.2011.11.055

18. Stam, C.J. and van Dijk, B.W. (2002) Synchronization Likelihood: An Unbiased Measure of Generalized Synchronization in Multivariate Data Sets. Physica D: Nonlinear Phenomena, 163, 236-251. https://doi.org/10.1016/S0167-2789(01)00386-4

19. Montez, T., Linkenkaer-Hansen, K., van Dijk, B.W. and Stam, C.J. (2006) Synchronization Likelihood with Explicit Time-Frequency Priors. NeuroImage, 33, 1117-1125. https://doi.org/10.1016/j.neuroimage.2006.06.066

20. Newman, M.E.J. (2006) Modularity and Community Structure in Networks. Proceedings of the National Academy Sciences, 103, 8577-8582. https://doi.org/10.1073/pnas.0601602103

21. Smith, E.E. and Jonides, J. (1999) Storage and Executive Processes in the Frontal Lobes. Science, 283, 657-1661. https://doi.org/10.1126/science.283.5408.1657 
22. Levy-Cooperman, N., Burhan, A.M., Rafi-Tari, S., Kusano, M., Ramirez, J., Caldwell, C. and Black, S.E. (2008) Frontal Lobe Hypoperfusion and Depressive Symptoms in Alzheimer Disease. Journal of Psychiatry Neuroscience, 33, 218-226.

23. Rossini, P.M., Buscema, M., Capriotti, M. and Grossi, E. (2008) Is It Possible to Automatically Distinguish Resting EEG Data of Normal Elderly vs. Mild Cognitive Impairment Subjects with High Degree of Accuracy? Clinical Neurophysiology, 119, 1534-1545. https://doi.org/10.1016/j.clinph.2008.03.026

24. Buscema, M., Vernieri, F., Massini, G. and Scrascia, F. (2015) An Improved I-FAST System for the Diagnosis of Alzheimer's Disease from Unprocessed Electroencephalograms by Using Robust Invariant Features. Artificial Intelligence in Medicine, 64, 59-74. https://doi.org/10.1016/j.artmed.2015.03.003

25. Buscema, P.M., Rossini, P., Babiloni, C. and Grossi, E. (2007) The IFAST Model, a Novel Parallel Nonlinear EEG Analysis Technique, Distinguishes Mild Cognitive Impairment and Alzheimer's Disease Patients with High Degree of Accuracy. Artificial Intelligence in Medicine, 40, 127-141. https://doi.org/10.1016/j.artmed.2007.02.006

26. Buscema, P.M., Grossi, E., Capriotti, M., Babiloni, C. and Possini, P. (2010) The IFAST. Model Allows the Prediction of Conversion to Alzheimer Disease in Patients with Mild Cognitive Impairment with High Degree of Accuracy. Current Alzheimer Research, 7, 173-187. https://doi.org/10.2174/156720510790691137

27. Trambaiollo, L.R., Lorena, A.C., Fraga, F.J., Kanda, P.A.M., Anghinah, R. and Nitrini, R. (2011) Improving Alzheimer's Disease Diagnosis with Machine Learning Techniques. Clinical EEG Neuroscience, 42, 160-165. https://doi.org/10.1177/155005941104200304

28. McBride, J.C., Zhao, X., Munro, N.B., Smith, C.D., Jicha, G., Hively, L., Broster, L., Schmitt, F.A., Kryscio, R.J. and Jiang, Y. (2014) Spectral and Complexity Analysis of Scalp EEG Characteristics for Mild Cognitive Impairment and Early Alzheimer's Disease. Computer Methods and Programs in Biomedicine, 114, 153-163. https://doi.org/10.1016/j.cmpb.2014.01.019

29. Braddley, A.D. (2000) The Episodic Buffer: A New Component of Working Memory? Trends in Cognitive Science, 4, 417-423. https://doi.org/10.1016/S1364-6613(00)01538-2

30. Buckner, R.L., Andrews-Hanna, J.R. and Schacter, D.L. (2008) The Brain's Default Network: Anatomy, Function, and Relevance to Disease. Annals of the New York Academy of Sciences, 1124, 1-38. https://doi.org/10.1196/annals.1440.011

31. Summerfield, J.J., Hassabis, D. and Maguire, E.A. (2010) Differential Engagement of Brain Regions within a "Core" Network during Scene Construction. Neuropsychologia, 48, 1501-1509.

https://doi.org/10.1016/j.neuropsychologia.2010.01.022

32. Sadaghiani, S., Scheeringa, R., Lehongre, K., Morillon, B., Giraud, A.-L. and Kleinschmidt, A. (2012) Alpha-Band Phase Synchrony is Related to Activity in the Fronto-Parietal Adaptive Control Network. Journal of Neuroscience, 32, 14305-14310. https://doi.org/10.1523/JNEUROSCI.1358-12.2012

33. Damoiseaux, J.S., Rombouts, S.A.R.B., Barkhof, F., Scheltens, P., Stam, C.J., Smith, S.M. and Beckmann, C.F. (2006) Consistent Resting-State Networks across Healthy Subjects. Proceedings of the National Academy of Sciences of the United States of America, 103, 13848-13853. https://doi.org/10.1073/pnas.0601417103

34. Deco, G. and Corbetta, M. (2011) The Dynamical Balance of the Brain at Rest. Neuroscientist, 17, $107-123$. https://doi.org/10.1177/1073858409354384

35. De Luca, M., Beckmann, C.F., De Stephano, N., Matthews, P.M. and Smith, S.M. (2006) fMRI Resting State Networks Define Distinct Modes of Long-Distance Interactions in the Human Brain. Neuroimage, 29, 1359-1367. https://doi.org/10.1016/j.neuroimage.2005.08.035

36. Doucet, G., Naveau, M., Petit, L., Delcroix, N., Zago, L., Crivello, F., Jobard, G., Tzourio-Mazoyer, N., Mazoyer, B., Mellet, E. and Joliot, M. (2011) Brain Activity at Rest: A Multi-Scale Hierarchical Functional Organization. Journal of Neurophysiology, 105, 2753-2763. https://doi.org/10.1152/jn.00895.2010 
37. Van der Heuvel, M.P. and Hulshoff Pol, H.E. (2010) Exploring the Brain Network: A Review on Resting-State fMRI Functional Connectivity. European Neuropsychopharmacology, 20, 519-534. https://doi.org/10.1016/j.euroneuro.2010.03.008

38. Fox, M.D., Snyder, A.Z., Vincent, J.L., Corbetta, M., Van Essen, D.C. and Raichle, M.E. (2005) The Human Brain Is Intrinsically Organized into Dynamic, Anticorrelated Functional Networks. Proceedings of the National Academy of Science of the United States of America, 102, 9673-9678. https://doi.org/10.1073/pnas.0504136102

39. Greicius, M.D., Krasnow, B., Reiss, A.L. and Menon, V. (2003) Functional Connectivity in the Resting Brain: A Network Analysis of the Default Mode Hypothesis. Proceedings of the National Academy Science, 100, 253-258. https://doi.org/10.1073/pnas.0135058100

40. Hampson, M., Driesen, N.R., Skudlarski, P., Gore, J.C. and Constable, R.T. (2006) Brain Connectivity Related to Working Memory Performance. Journal of Neuroscience, 26, 13338-13343. https://doi.org/10.1523/JNEUROSCI.3408-06.2006

41. Pyka, M., Beckmann, C.F., Schöning, S., Hauke, S., Heider, D., Kugel, H., Arolt, V. and Konrad, C. (2009) Impact of Working Memory Load on fMRI Resting State Pattern in Subsequent Resting Phases. PLoS ONE, 4, e7198. https://doi.org/10.1371/journal.pone.0007198

42. Sridharan, D., Levitin, D.J. and Menon, V. (2008) A Critical Role for the Right Fronto-Insular Cortex in Switching between Central-Executive and Default Mode Networks. Proceedings of the National Academy Sciences, 105, 12569-12574. https://doi.org/10.1073/pnas.0800005105

43. Tomasi, D., Ernst, T., Caparelli, E.C. and Chang, L. (2006) Common Deactivation Patterns during Working Memory and Visual Attention Tasks: An Intrasubject fMRI Study at 4 Tesla. Human Brain Mapping, 27, 694-705. https://doi.org/10.1002/hbm.20211

44. Vincent, J.L., Kahn, I., Snyder, A.Z., Raichle, M.E. and Buckner, R.L. (2008) Evidence for a Frontoparietal Control System Revealed by Intrinsic Functional Connectivity. Journal of Neurophysiology, 100, 3328-3342. https://doi.org/10.1152/jn.90355.2008

45. Moosmann, M., Ritter, P., Krastel, I., Brink, A., Thees, S., Blankenburg, F., Taskin, B., Obrig, H. and Villringer, A. (2003) Correlates of Alpha Rhythm in Functional Magnetic Resonance Imaging and near Infrared Spectroscopy. Neuroimage, 20, 145-158. https://doi.org/10.1016/S1053-8119(03)00344-6

46. Hastie, T.J. and Pregibon, D. (1992) Generalized Linear Models. In: Chambers, J.M. and Hastie, T.J., Eds., Statistical Models in S, Wadsworth \& Brooks/Cole, Pacific Grove, Chapter 6. 